\title{
Numerical methods for the TSD equation in conservation law form
}

\author{
Natalie J.T. Phillips John A. Gear Hing Hung*
}

(Received 7 August 2000)

\begin{abstract}
The Transonic Small Disturbance (TSD) Equation is a common model equation for describing subsonic and supersonic flow close to the local speed of sound (transonic). In transonic flow there is an embedded region of locally supersonic flow inside an otherwise subsonic flow. The supersonic region is usually terminated by a shock discontinuity. The success of a numerical scheme for transonic flow
\end{abstract}

*Department of Mathematics, RMIT University, Melbourne, Victoria 3001, AustraliA. mailto:n.phillips@rmit.edu.au

${ }^{0}$ See http://anziamj. austms .org.au/V42/CTAC99/Phil for this article and ancillary services, (c) Austral. Mathematical Soc. 2000. Published 27 Nov 2000. 
prediction depends on its capability of capturing all the flow details and non-linearities including sharp shock profiles without oscillations near the shock.

Most of the important phenomena in the TSD equation occur in the stream-wise direction. The nonlinearity and changes in the region of influence depend only on the stream-wise derivation. A suitable one-dimensional model equation derived from the TSD Equation is used. The one-dimensional equation is written in conservation law form. This nonlinear system of equations models the transition from supersonic to subsonic flow.

In the numerical calculations the discretised problem is treated as a series of Riemann problems. We will investigate various techniques for solving these Riemann problems. It will be shown that the techniques do not allow non-physical expansion shocks to develop and that the techniques smooth out expansion shocks when these non-physical shocks are present in the initial velocity profile. A comparison will be made between the schemes based on the sharpness of the resulting shock profiles.

\section{Contents}

1 Introduction

C1158

2 Test Problem

C1161 
3 System of Equations

C1163

4 MUSCL-Hancock Scheme

C1168

5 WAF

C1171

References

C1177

\section{Introduction}

The Transonic Small Disturbance (TSD) Equation for the perturbation velocity potential $\phi$, see [1], is

$$
\begin{aligned}
-M^{2} \phi_{t t} & -2 M^{2} \frac{\partial}{\partial t}\left(\phi_{x}-\bar{w}\right)-\frac{\beta^{2}}{2 \bar{u}} \frac{\partial}{\partial x}\left(\phi_{x}-\bar{w}\right)^{2} \\
& +\phi_{y y}+\phi_{z z}=0,
\end{aligned}
$$

where $\beta^{2}=1-M^{2}, \bar{w}=\beta^{2} /\left[M^{2}(1+\gamma)\right], M$ is the free stream Mach number and $\gamma$ is the ratio of specific heats. Here $x, y, z$ represent a nondimensional coordinate system and $t$ is the non-dimensional time variable. In non-dimensional terms the fluid velocity is defined by $\mathbf{v}=\nabla(x+\phi)$. In transonic flow there is an embedded region of locally supersonic flow $\left(\phi_{x}>\bar{w}\right)$ inside an otherwise subsonic flow $\left(\phi_{x}<\bar{w}\right)$. The supersonic region is usually terminated by a shock discontinuity. 
Most of the important phenomena in the TSD Equation occur in the stream-wise, $x$, direction. The nonlinearity and the switch in the difference formulas depend only on the stream-wise derivative. If we ignore the $y$ and $z$ dependence in (1), and substitute $\psi=\beta^{2}(\phi-\bar{w} x) /\left(2 \bar{w} M^{2}\right)$, a suitable one-dimensional model equation is

$$
\frac{\partial}{\partial t}\left(\psi_{t}+2 \psi_{x}\right)+\frac{\partial}{\partial x}\left(\psi_{x}^{2}\right)=0 .
$$

Note that the flow is locally supersonic when $\psi_{x}>0$ and subsonic when $\psi_{x}<0$. Also if the solution is time independent then $\left(\psi_{x}^{2}\right)_{x}=0$ and $\psi_{x}$ is piecewise constant.

In conservation law form Equation (2) can be written as

$$
\mathbf{u}_{t}+\mathbf{f}(\mathbf{u})_{x}=\left(\begin{array}{l}
u \\
v
\end{array}\right)_{t}+\left(\begin{array}{c}
a u-v \\
u^{2}+(2-a) v-a(2-a) u
\end{array}\right)_{x}=0
$$

where $u=\psi_{x}$ and $v=\psi_{t}+a \psi_{x}$. Usually $0 \leq a \leq 2$. Alternatively we can write $\mathbf{u}_{t}+A \mathbf{u}_{x}=0$, where $A$ is the Jacobian of $\mathbf{f}(\mathbf{u})$ given by

$$
A=\left(\begin{array}{cc}
a & -1 \\
2 u-a(2-a) & 2-a
\end{array}\right) .
$$

In [4] we looked at modelling the scalar form of the TSD Equation, Equation (2). The schemes were found to have sharp shocks and most eliminated nonphysical shocks. We looked at second order extensions to these schemes 
in [5] and also investigated first order schemes to solve the TSD Equation in conservation law form, Equation (3). In what follows we will be looking at second order schemes to solve the TSD Equation in conservation law form.

From the Rankine Hugoniot shock jump condition [3] we have

$$
\mathbf{f}\left(\mathbf{u}_{r}\right)-\mathbf{f}\left(\mathbf{u}_{l}\right)=s\left(\mathbf{u}_{r}-\mathbf{u}_{l}\right)
$$

where $\mathrm{s}$ is the shock speed and where $\mathbf{u}_{l}$ and $\mathbf{u}_{r}$ are the value of $\mathbf{u}$ to the left and right of the shock respectively. This gives us that $s=\Delta \psi_{t} / \Delta \psi_{x}$ and $s=1 \pm \sqrt{1-2 \bar{u}}$ where $\Delta \psi_{t}$ is the change in $\psi_{t}$ across the shock and $\bar{u}=\left(u_{r}+u_{l}\right) / 2$. For a time independent solution $s=0$ so $u_{l}=-u_{r}$. For a solution which satisfies entropy we must have $u_{l}>u_{r}$.

From Equations (2) and (4) the characteristics are $\partial x / \partial t=1 \pm \sqrt{1-2 \psi_{x}}$. When $\psi_{x}>0$ the flow is supersonic and both the characteristics have positive gradients (assuming $\psi_{x}<1 / 2$ ), indicating that the domain of dependence is upstream and hence upwind differencing should be employed. Whereas for $\psi_{x}<0$ the domain of dependence is central and $x$-derivatives need to be evaluated using central difference approximations. A basic requirement of any type-dependent differencing is that it changes smoothly from central to upwind differencing as the flow changes from subsonic to supersonic [2]. This guarantees that non-physical decompression shocks will not develop. It also ensures that the shock jump conditions are satisfied at the re-compression shock (supersonic to subsonic), (see [6]). 


\section{Test Problem}

The test problem was chosen to highlight different aspects of the techniques employed to solve the one dimensional model problem. The most important attributes of any scheme were deemed to be its ability to remove any nonphysical shock, also the sharpness of any physical shock in the solution. These attributes are used to judge a scheme's success.

In the numerical experiments the initial state was defined as

$$
\psi_{x}(x, 0)= \begin{cases}0.4, & 0 \leq x \leq \frac{1}{2}, 1 \leq x \leq \frac{3}{2} \\ -0.4, & \frac{1}{2} \leq x \leq 1, \frac{3}{2} \leq x \leq 2\end{cases}
$$

In transonic flow we expect both supersonic and subsonic flow. In one dimension a steady state for a transonic flow must be supersonic upstream and subsonic downstream with the two states joined by a shock. For this reason both the test problems are supersonic at the upstream boundary and subsonic at the down stream boundary. The test problem, (see Figure 1), was designed to include an entropy violating, or non-physical, shock. For any scheme to be useful it must prevent these non-physical shocks from forming and eliminate any that are present. This test problem has three shocks where the flow changes between supersonic and subsonic. The second shock is non-physical, the flow changes from subsonic to supersonic. In a successful scheme this shock will be smoothed out, and the three shocks merge into one at steady state. 


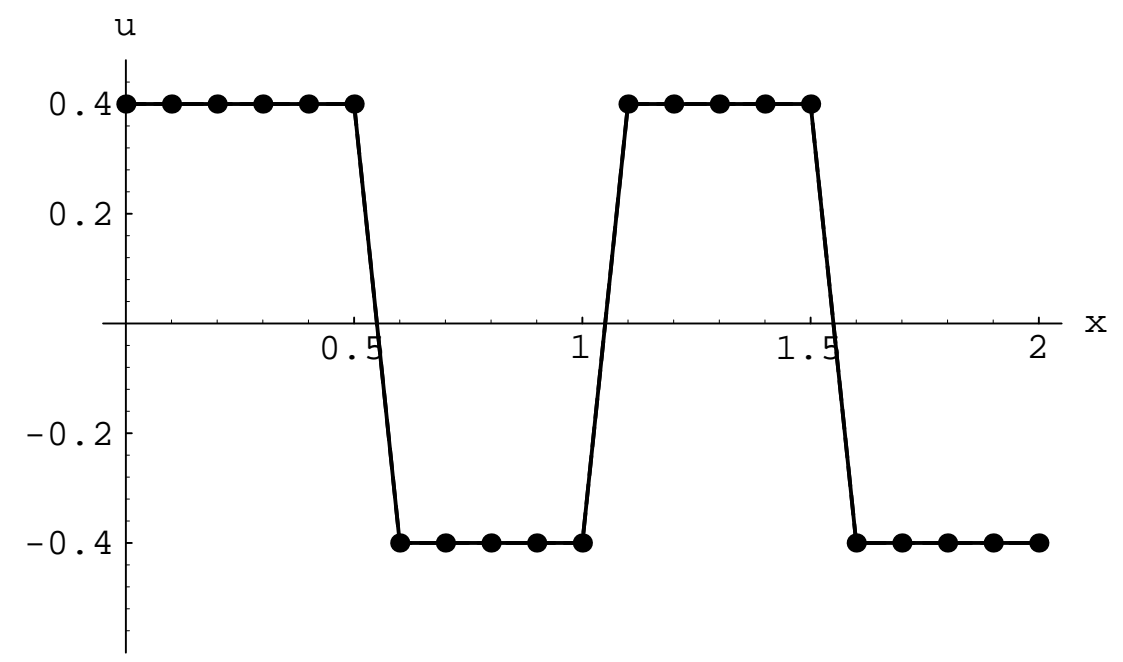

Figure 1: Test problem. 


\section{System of Equations}

Here we are interested in the numerical solution of the 1-D model problem in conservation law form, see Equation (3). There has been much work done on these types of equations [8]. The TSD Equation has typically been solved in scalar form. In previous papers we have investigated numerical schemes for the scalar form of the one-dimensional test problem, Equation (2), (see [4, 5]). In the scalar form of the TSD equation the transformed partial differential equation, solved numerically, depended upon the structure of the grid transformation. The main advantage of writing the TSD equation in conservation law form is that the solution process is independent of the grid transformation. The nature of the differencing, whether central or backward differencing, follows naturally from the eigenvalues of the system. The entropy can be related to the eigenvalues around a shock.

The numerical method we have used for this system of equations discretises the $x$-axis and takes each adjacent cell as one in a series of Riemann problems. The difference scheme is

$$
\mathbf{U}_{i}^{n+1}=\mathbf{U}_{i}^{n}-\frac{\Delta t}{\Delta x}\left[\mathbf{F}\left(\mathbf{U}_{i+1}^{n}, \mathbf{U}_{i}^{n}\right)-\mathbf{F}\left(\mathbf{U}_{i}^{n}, \mathbf{U}_{i-1}^{n}\right)\right]
$$

where we define $\mathbf{U}_{i}$ as the average of $\mathbf{u}_{i}$ across the interval $\left(x_{i-1 / 2}, x_{i+1 / 2}\right)$, $\mathbf{U}_{i}=1 / \Delta t \int_{x_{i}-1 / 2}^{x_{i}+1 / 2} \mathbf{u} d x$, and we define $\mathbf{F}\left(\mathbf{U}_{r}, \mathbf{U}_{l}\right)$ as the numerical flux at the boundary of left and right states $\mathbf{U}_{l}$ and $\mathbf{U}_{r}$ respectively. At the upstream 
and downstream boundaries we use the following transmissive conditions

$$
\begin{array}{cc}
\mathbf{U}_{0}=\mathbf{U}_{1} & \mathbf{U}_{-1}=\mathbf{U}_{2} \\
\mathbf{U}_{N+1}=\mathbf{U}_{N} & \mathbf{U}_{N+2}=\mathbf{U}_{N-1} .
\end{array}
$$

A popular method for solving conservation equations was suggested by Roe [7]. Rather than solving the non-linear problem an approximate linear flux is used where $\mathbf{f}(\mathbf{u}) \approx \tilde{\mathbf{f}}(\mathbf{u})=\tilde{A} \mathbf{u}$. This approximate Jacobian $\tilde{A}$ will depend on $\mathbf{u}_{l}$ and $\mathbf{u}_{r}$, the respective left and right states of $\mathbf{u}$, and will comply with the following conditions:

1. $\tilde{A}\left(\mathbf{u}_{l}, \mathbf{u}_{r}\right)\left(\mathbf{u}_{r}-\mathbf{u}_{l}\right)=\mathbf{f}\left(\mathbf{u}_{r}\right)-\mathbf{f}\left(\mathbf{u}_{l}\right)$,

2. $\tilde{A}\left(\mathbf{u}_{l}, \mathbf{u}_{r}\right)$ is diagonalisable with real eigenvalues,

3. $\tilde{A}\left(\mathbf{u}_{l}, \mathbf{u}_{r}\right) \rightarrow \mathbf{f}^{\prime}(\overline{\mathbf{u}})$ smoothly as $\mathbf{u}_{l}, \mathbf{u}_{r} \rightarrow \overline{\mathbf{u}}$.

Replacing $\mathbf{u}$ by the average $\left(\mathbf{U}_{l}+\mathbf{U}_{r}\right) / 2$ gives us our approximate $\tilde{A}$, there may be other suitable approximations. Condition 1 can easily be shown to be satisfied. The eigenvalues become $\tilde{\lambda}=1 \pm \sqrt{1-\left(u_{l}+u_{r}\right)}$, and condition 2 is satisfied when $u_{l}+u_{r}<1$ so $u_{\max }<1 / 2$. Condition 3 and Equation (5) implies that the shock speed must be an eigenvector of $\tilde{A}$.

The flux for Roe's method comes from examining the integral form of 
Equation (7) over $x \in[-M, M]$ and $t \in[0,1]$

$$
\int_{-M}^{M} \hat{\mathbf{U}}(\xi) d \xi=M\left(\mathbf{U}_{L}+\mathbf{U}_{R}\right)+\mathbf{f}\left(\mathbf{U}_{L}\right)-\mathbf{f}\left(\mathbf{U}_{R}\right),
$$

for $M$ sufficiently large, where $\hat{\mathbf{U}}$ is the solution of the linearised problem. If $\mathbf{F}\left(\mathbf{U}_{L}, \mathbf{U}_{R}\right)$ is the numerical flux along $x=0$ then

$$
\mathbf{F}\left(\mathbf{U}_{L}, \mathbf{U}_{R}\right)=M \mathbf{U}_{L}+\mathbf{f}\left(\mathbf{U}_{L}\right)-\int_{-M}^{0} \hat{\mathbf{U}}(\xi) d \xi .
$$

To be conservative, the new flux must satisfy $\tilde{\mathbf{f}}\left(\mathbf{U}_{R}\right)-\tilde{\mathbf{f}}\left(\mathbf{U}_{L}\right)=\mathbf{f}\left(\mathbf{U}_{R}\right)-\mathbf{f}\left(\mathbf{U}_{L}\right)$, and Equation (8). The flux from the linearised system at $x=0$ is $\tilde{\mathbf{f}}(\hat{\mathbf{U}}(0))$ so

$$
\int_{-M}^{0} \hat{\mathbf{U}}(\xi) d \xi=M \mathbf{U}_{L}+\tilde{\mathbf{f}}\left(\mathbf{U}_{L}\right)-\tilde{\mathbf{f}}(\overline{\mathbf{U}}(0)) .
$$

Combining Equation (9) and (10) we find the inter-cell flux is $\mathbf{F}\left(\mathbf{U}_{L}, \mathbf{U}_{R}\right)=$ $\tilde{\mathbf{f}}(\overline{\mathbf{U}}(0))+\mathbf{f}\left(\mathbf{U}_{L}\right)-\tilde{\mathbf{f}}\left(\mathbf{U}_{L}\right)$. Rewriting using $\tilde{\mathbf{f}}(\mathbf{U})=\tilde{A} \mathbf{U}$ and $\overline{\mathbf{U}}(0)=\mathbf{U}_{L}+$ $\sum_{\tilde{\lambda}_{p}<0} \alpha_{p} \tilde{r}_{p}$ we find that

$$
\mathbf{F}\left(\mathbf{U}_{L}, \mathbf{U}_{R}\right)=\mathbf{f}\left(\mathbf{U}_{L}\right)+\sum_{\tilde{\lambda}_{p}<0} \tilde{\lambda}_{p} \alpha_{p} \tilde{r}_{p}
$$

where $\tilde{\lambda}_{p}, \tilde{\mathbf{r}}_{p}$ are the eigenvalues and eigenvectors of $\tilde{A}$ respectively and $\alpha_{p}$ is defined by $\mathbf{U}_{r}-\mathbf{U}_{l}=\sum \alpha_{p} \tilde{\mathbf{r}}_{p}$. The inter-cell flux in this form, for right travelling supersonic flow, gives the flux $\mathbf{f}\left(\mathbf{U}_{L}\right)$ as expected. 
This method produces a solution which has a sharp shock profile but unfortunately admits entropy violating discontinuities. A correction by Harten and Hyman [3], eliminates the entropy violating shocks without smearing the re-compression shock. A shock with characteristics heading out of it is not stable and any amount of viscosity (in real situations viscosity plays a role in the region of a shock) would smear the solution out into a rarefaction wave. When the characteristics are heading into the shock, the shock is stable. Characteristics point out of a shock when $\lambda_{p l}<s<\lambda_{p r}$, where $\lambda_{p l}=\lambda_{p}\left(\mathbf{U}_{l}\right)$. Since Roe's method only looks at the solution along the cell divides we need only to insert a rarefaction wave for $\lambda_{p l}<0<\lambda_{p r}$. The correction by Harten and Hyman prevents subsonic to supersonic shocks from forming and smooths them out when they are present. The correction is achieved by inserting an intermediate value of $\mathbf{U}, \mathbf{U}^{*}$, between the characteristic speeds $\lambda_{p l}, \lambda_{p r}$. The value of $\mathbf{U}^{*}$ is chosen to conserve $\mathbf{u}$ in that interval. So $\mathbf{U}^{*}$ must agree with,

$$
\left(\lambda_{p r}-\lambda_{p l}\right) \mathbf{U}^{*}=\left(\tilde{\lambda}_{p}-\lambda_{p l}\right) \mathbf{U}_{p l}+\left(\lambda_{p r}-\tilde{\lambda}_{p}\right) \mathbf{U}_{p r},
$$

$\mathbf{U}_{p l}$ and $\mathbf{U}_{p r}$ are the values of $\mathbf{U}$ to the left and right of the characteristic $\tilde{\lambda}_{p}$, not necessarily the left and right states of the Riemann problem. Equation (12) can be satisfied by replacing $\tilde{\lambda}_{p}$ by $\tilde{\lambda}_{p}\left(\lambda_{p r}-\tilde{\lambda}_{p}\right) /\left(\lambda_{p r}-\lambda_{p l}\right)$ in Equation (11), whenever the inequality $\lambda_{p l}<0<\lambda_{p r}$ holds.

Figure 2 shows the result of the Roe scheme with the Harten and Hyman correction. The initial state is given by Equation (6). Here $\Delta t=0.025$ and $\Delta x=0.5$. To reach steady-state 128 time steps were performed. Notice 

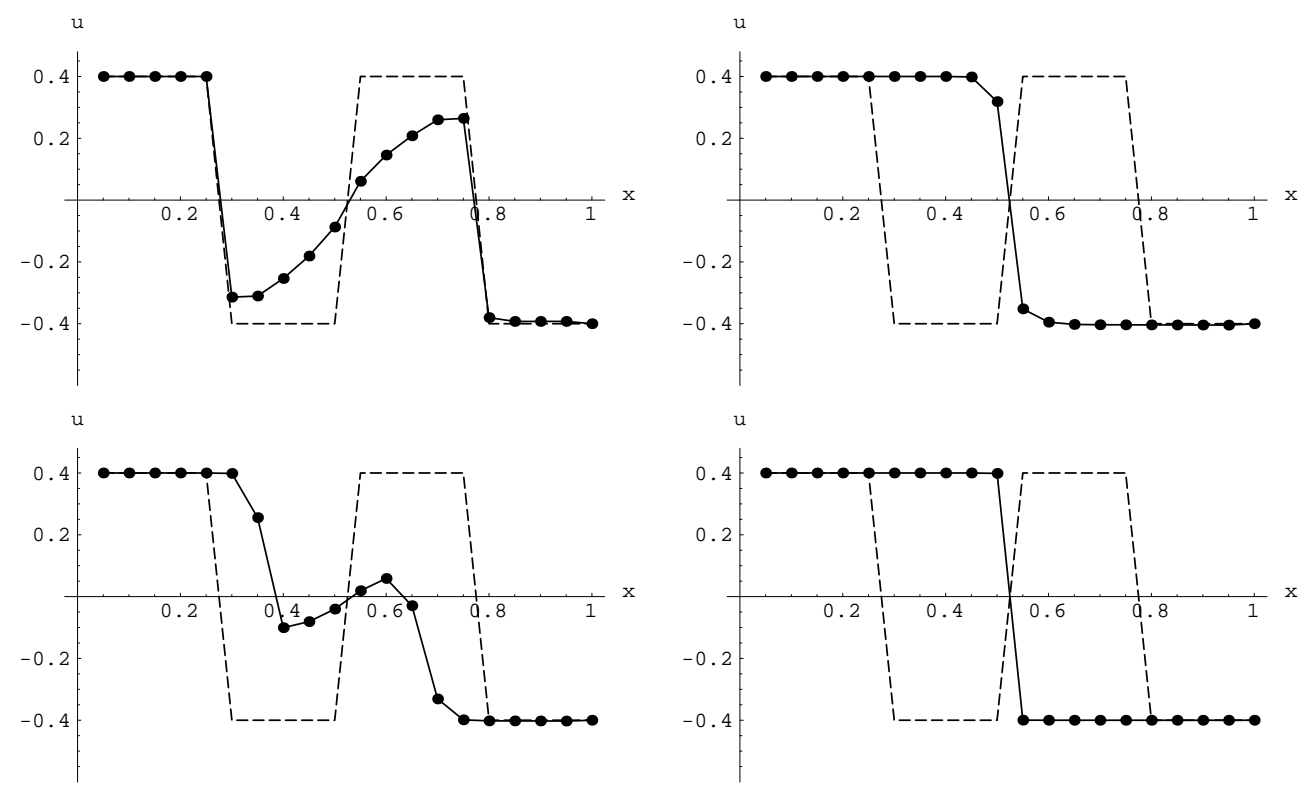

Figure 2: Roe scheme with Harten and Hyman correction at time steps $t=20, t=60, t=100$ and when converged at $t=128$, Initial condition (dotted) and steady-state (continuous). 
that the final solution has a sharp shock profile and that the non-physical expansion shock in the initial state has been eliminated.

\section{MUSCL-Hancock Scheme}

The MUSCL-Hancock [9, 10, 8] and subsequent WAF [8] methods are second order extensions to Roe's method. The MUSCL-Hancock Scheme (Monotonic Upstream-Centred Scheme for Conservation Laws) achieves second order accuracy by taking a piecewise linear approach to find the intercell fluxes. The initial data is converted to piecewise linear using MINMOD to find the slope.

Using the MINMOD slope limiter the MUSCL-Hancock scheme becomes Total Variation Diminishing (TVD). A requirement of TVD limiters is the value of $\mathbf{U}_{i}^{n+1}$ will be restricted to be between the values of $\mathbf{U}_{i-s}^{n}, \mathbf{U}_{i}^{n}$ where $s$ is $\operatorname{sign}(\lambda)$, that is the value of $\mathbf{U}$ at the next time step is between the previous value of $\mathbf{U}$ and the value of $\mathbf{U}$ upstream. This limiting does not allow spurious oscillations to occur. The MINMOD technique looks at the difference between two nodes. The slope is given by,

$$
\Delta_{i}=\left(\operatorname{sign} \Delta_{i-1 / 2}+\operatorname{sign} \Delta_{i+1 / 2}\right)\left(\min \left[\operatorname{abs}\left(\Delta_{i-1 / 2}\right), \operatorname{abs}\left(\Delta_{i+1 / 2}\right)\right]\right) / 2,
$$

where $\Delta_{i-1 / 2}=\mathbf{U}_{i}-\mathbf{U}_{i-1}$. If the neighbouring differences have the same sign then MINMOD gives the smallest of the neighbouring slopes. If the neighbouring differences have opposite signs then MINMOD gives zero slope. Using 
the new slope the value of $\mathbf{U}$ is calculated for the left and right boundaries of the cell. So $\overline{\mathbf{U}}_{i}^{L}=\mathbf{U}_{i}-\Delta_{i} / 2$ and $\overline{\mathbf{U}}_{i}^{R}=\mathbf{U}_{i}+\Delta_{i} / 2$.

A new value at each end of the interval is generated by evolving the solution, staying within the cell, by half a time step.

$$
\mathbf{U}_{i}^{L / R}=\mathbf{U}_{i}^{L / R}+\Delta t /(2 \Delta x)\left(\mathbf{F}\left(\mathbf{U}_{i}^{L}\right)-\mathbf{F}\left(\mathbf{U}_{i}^{R}\right)\right),
$$

The flux at the boundary is now calculated with the new values of $\mathbf{U}$ at the ends of the cells as adjacent values of a Riemann Problem. For example at the boundary $x_{i-1 / 2}$ the left and right values for the Riemann problem are $\overline{\mathbf{U}}_{i-1}^{R}$ and $\overline{\mathbf{U}}_{i}^{L}$ respectively with the new found $\mathbf{U}_{L}$ and $\mathbf{U}_{R}$. The Riemann problem is solved using the Roe's method shown earlier.

Figure 3 shows the result of using the MUSCL-Hancock scheme on the test problem. The scheme converges in 122 time steps. Note in all test cases $\Delta t=0.025$ and $\Delta x=0.05$.

A problem that is often encountered with many second order schemes is the oscillations that are produced near regions of high variation such as at the shock. The MUSCL-Hancock method does not suffer this problem due to the use of the TVD slope limiter MINMOD. It can also be seen that the intermediate solution has a smooth subsonic to supersonic expansion wave. 

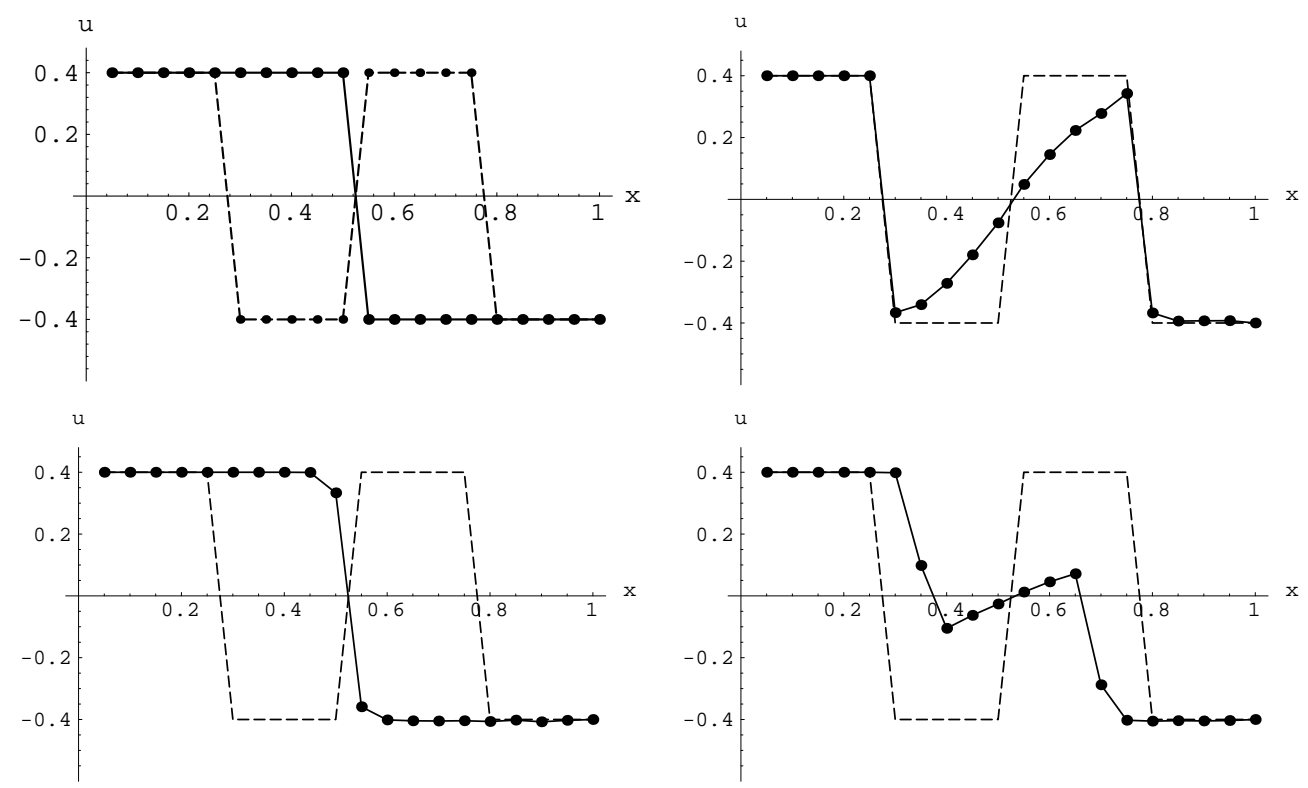

FiguRE 3: MUSCL-Hancock scheme with at time steps $t=20, t=60, t=100$ and when converged at $t=122$. 


\section{WAF}

The weighted average flux (WAF) [8] scheme is a second order scheme for hyperbolic systems of equations. The WAF method, as its name might suggest, takes an average of either the state $\mathbf{U}$ or the flux between two adjacent cells at the half time step. We have taken the approach of finding the average state of $\mathbf{U}$ across this interval rather than finding the average flux as it involves less calculation. The weighted average state of $\mathbf{U}$, denoted by $\overline{\mathbf{U}}$ in integral form, is given by

$$
\overline{\mathbf{U}}_{i+1 / 2}=\frac{1}{\Delta x} \int_{-\Delta x / 2}^{\Delta x / 2} \mathbf{U}_{i+1 / 2}(x, \Delta t / 2) d x,
$$

This integral is evaluated using constant states of $\mathbf{U}$ separated by two wave speeds. The wave speeds are found using the approximate eigenvalues from Roe's method. The solution at $\Delta t / 2$ is made up of three states of $\mathbf{U}$, $\mathbf{U}^{(1)}, \mathbf{U}^{(2)}$ and $\mathbf{U}^{(3)}$. $\mathbf{U}^{(1)}$ and $\mathbf{U}^{(3)}$ are given by the left and right state of the Riemann problem $\mathbf{U}_{i}$ and $\mathbf{U}_{i+1}$ respectively while $\mathbf{U}^{(2)}=\mathbf{U}_{i}+\alpha_{1} \tilde{r}_{1}$. The average state is $\overline{\mathbf{U}}_{i+1 / 2}=\sum_{k}^{N} \beta_{k} \mathbf{U}_{i+1 / 2}^{(k)}$, where $\beta_{k}$ is the proportion, weight, of $\mathbf{U}^{(k)}$ taken from the solution of the Riemann problem thus

$$
\overline{\mathbf{U}}_{i+1 / 2}=\left(1-\frac{\Delta t}{\Delta x} \tilde{\lambda}_{1}\right) \mathbf{U}^{(1)}+\frac{\Delta t}{\Delta x}\left(\tilde{\lambda}_{2}-\tilde{\lambda}_{1}\right) \mathbf{U}^{(2)}+\left(1-\frac{\Delta t}{\Delta x} \tilde{\lambda}_{2}\right) \mathbf{U}^{(3)} .
$$

Using Roe's approximation, rarefaction waves do not need to be calculated, recall for the Harten-Hyman correction the value of $\mathbf{U}$ is conserved over the 
interval, therefore the average state, $\overline{\mathbf{U}}$, would not change. The flux is now calculated using the new average state giving $\mathbf{F}_{i+1 / 2}=\mathbf{F}\left(\overline{\mathbf{U}}_{\mathbf{i}+\mathbf{1} / \mathbf{2}}\right)$. This flux is used in Equation (7) to find $\mathbf{U}$ at the next time level.

The WAF method automatically deals with rarefaction waves by using the average state of $\mathbf{U}$ rather that the value of $\mathbf{U}$ along the cell divide. Unfortunately the same mechanism that smooths out nonphysical shocks also stops re-compression shocks from being sharp. The process of averaging U generates spurious oscillations near the shocks, this is not unexpected with second order schemes near regions of high variation. For the WAF method to be of any use when applying it to our problem the shock must be sharp. The WAF scheme did not behave well near the shocks or regions of high variation which can be expected considering the averaging nature of the method. Near the shock the method develops large spurious oscillations and subsequently diverges. This is a recognised problem with this method that is alleviated by taking a TVD approach by applying limiters to the solution at $u(t+1 / 2)$ across the jumps such as

$$
\overline{\mathbf{U}}=\left[\left(\mathbf{U}^{(1)}+\mathbf{U}^{(3)}\right)-\operatorname{sign}\left(c_{1}\right) \phi_{1}\left(\mathbf{U}^{(2)}-\mathbf{U}^{(1)}\right)-\operatorname{sign}(c 2) \phi_{2}\left(\mathbf{U}^{(3)}-\mathbf{U}^{(2)}\right)\right] / 2,
$$

$c_{1}$ and $c_{2}$ are the Courant number across the two characteristic waves, $\phi_{1}$, $\phi_{2}$ are the limiters used across each wave. The limiters that are used, are equivalent to the flux limiters of van Albada, $\phi_{v a}$, van Leer, $\phi_{v l}$, MINBEE, $\phi_{m a}$, and SUPERBEE, $\phi_{s a}$, given by Equations (13) through (16). The limiters are related to the flux limiters by $\phi(r)=1-(1-|c|) \psi(r)$, (see [8]) where $\psi$ is 

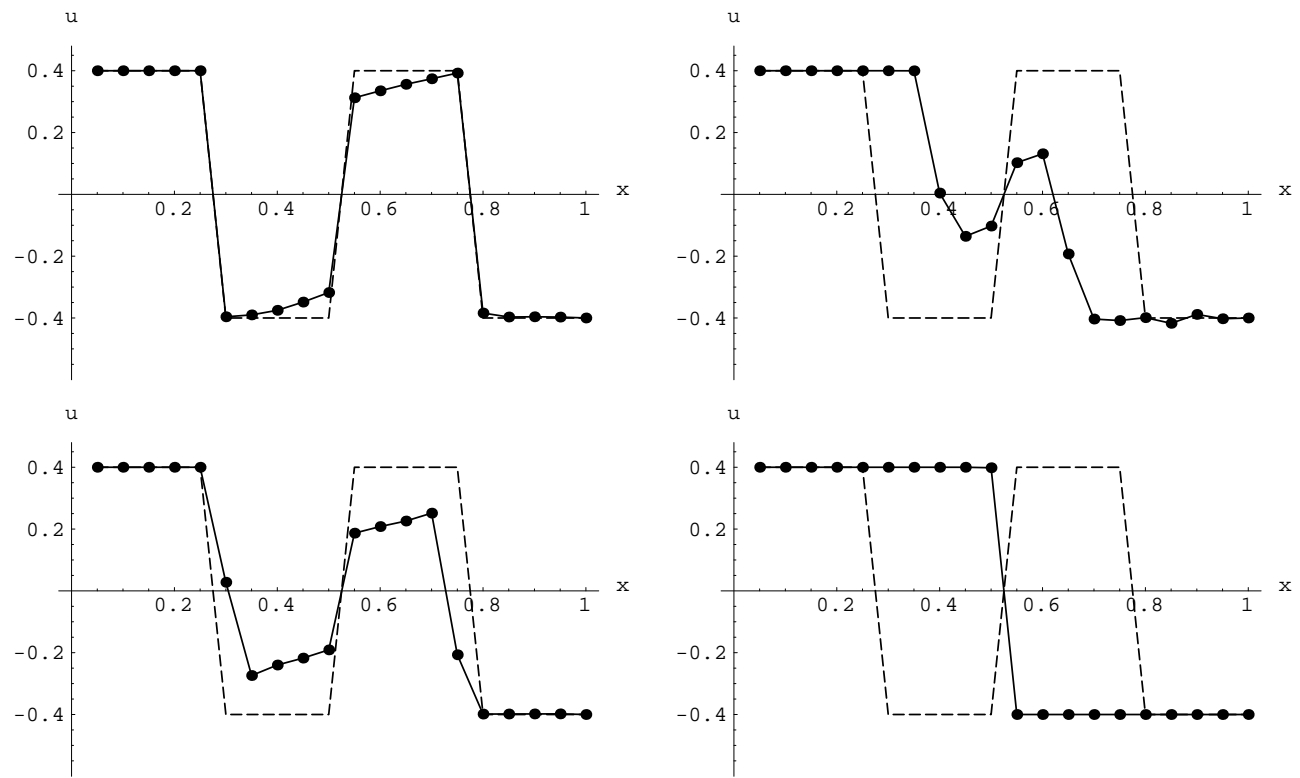

Figure 4: WAF scheme with van Albada's limiter at time steps $t=20, t=$ $60, t=100$ and when converged at $t=157$. 
the flux limiter.

$$
\begin{gathered}
\phi_{v a}(r,|c|)= \begin{cases}1 & \text { if } \quad r \leq 0, \\
1-\frac{(1-|c|) r(1+r)}{1+r^{2}} & \text { if } \quad r \geq 0 .\end{cases} \\
\phi_{v l}(r,|c|)= \begin{cases}1 & \text { if } \quad r \leq 0, \\
1-\frac{(1-|c|) 2 r}{1+r} & \text { if } \quad r \geq 0 .\end{cases} \\
\phi_{m a}(r,|c|)=\left\{\begin{array}{lll}
1 & \text { if } \quad r \leq 0, \\
1-(1-|c|) r & \text { if } \quad 0 \leq r \leq 1, \\
|c| & \text { if } \quad r \geq 1 .
\end{array}\right. \\
\phi_{s a}(r,|c|)=\left\{\begin{array}{lll}
1 & \text { if } \quad r \leq 0, \\
1-2(1-|c|) r & \text { if } & 0 \leq r \leq 1 / 2, \\
|c| & \text { if } & 1 / 2 \leq r \leq 1, \\
1-(1-|c|) r & \text { if } & 1 \leq r \leq 1, \\
2|c|-1 & \text { if } & r \geq 2 .
\end{array}\right.
\end{gathered}
$$

The limiters all change with the value of $r$ which is the ratio of upstream change in $\mathbf{u}$, over the local change.

$$
r_{i+1 / 2}= \begin{cases}\Delta_{i-1 / 2} / \Delta_{i+1 / 2} & \text { for } \tilde{\lambda}>0 \\ \Delta_{i+3 / 2} / \Delta_{i+1 / 2} & \text { for } \tilde{\lambda}<0 .\end{cases}
$$


The Courant number, c, is taken to be $\Delta t / \Delta x \tilde{\lambda}, \tilde{\lambda}$ is taken to be the wave speed. When there is a relatively smooth change $r \approx 1$ and $\phi \approx|c|$ giving the original second order WAF scheme. When $r \approx 0$, going into a region of high variation, $\phi \approx 1$ giving a first order upwind scheme which we know from Roe's method captures the shock well. When there is a change in the sign between the upwind and local difference, one is increasing while the other is decreasing giving values of $r<0$, then all of the limiters equal 1 resulting in a first order scheme.

The desired steady state was reached when applying each of the limiters to the WAF method. Regardless of the limiter used the entropy violating discontinuities were eliminated, and the shock in the final solution was sharp. Figure 4 and Figure 5 show the WAF scheme with van Albada limiter and van Leer limiter respectively. The limiters MINBEE and SUPERBEE are not shown as their output bears a strong resemblance to the limiters of van Albada and van Leer respectively. The van Leer limiter can be seen to converge faster than van Albada's, which is slower than Roe's method. It also has a smoother expansion in the intermediary solution.

Of all the second order methods the best scheme would appear to be the MUSCL scheme. This scheme had the fastest convergence. The entropy violating shock was quickly smoothed into a physically acceptable expansion fan. Almost as good was the WAF scheme with Van Leer's or the SUPERBEE limiter, which both took the same number of iterations to converge.

In future work with two and three dimensional problems a schemes han- 

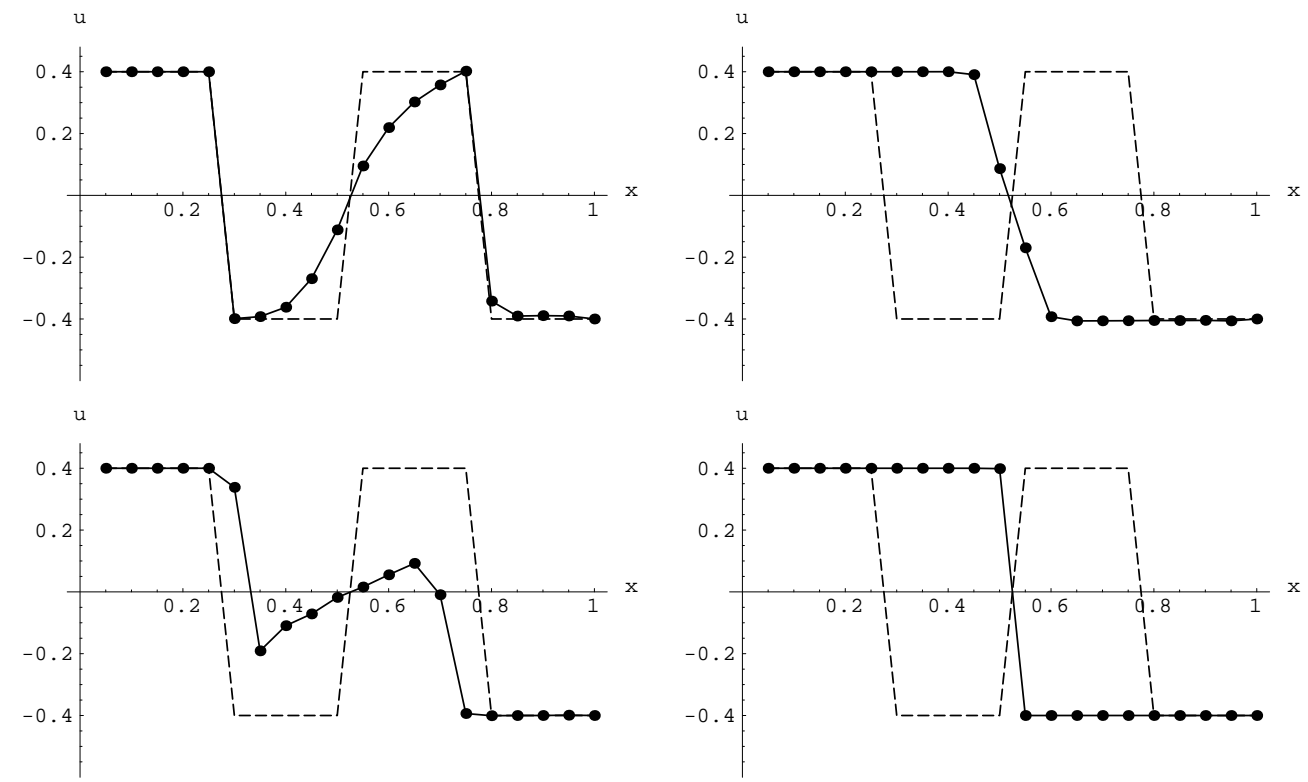

FiguRE 5: WAF scheme with van Leer's limiter at time steps $t=20, t=$ $60, t=100$ and when converged at $t=128$. 
dling of the rarefaction fan will be important. In these higher dimensions rarefaction fans will feature in the steady state solutions. The intermediate results of the WAF scheme with the Van Albada's or MINBEE limiter contain entropy violating shocks. This is different from other techniques investigated which smooth out this shock after only a few time time steps. This may make the WAF scheme with Van Albada's or MINBEE limiter unsuitable for the TSD equation with several dimensions.

\section{References}

[1] J.T. Batina. Efficient algorithm for solution of the unsteady transonic small-disturbance equation. J. Aircraft, 25:598-605, 1988. C1158

[2] B. Engquist and S. Osher. Stable and entropy satisfying approximations for transonic flow calculations. Mathematics of Computation, 34:45-75, 1980. C1160

[3] R.J. LeVeque. Numerical Methods for Conservation Laws. Birkhauser Verlag, 1990. C1160, C1166

[4] N.J.T. Phillips, J.A. Gear, and E. Ly. Type-dependent differencing schemes for transonic flow computation. In J. Noye, M.D. Teubner, and A. Gill, editors, Computational Techniques and Applications Conference: CTAC97, pages 537-544, Singapore 1998. World Scientific. C1159, C1163 
[5] N.J.T. Phillips, J.A. Gear, and E. Ly. Adaptive numerical methods for transonic flows with embedded shock discontinuities. In E.O. Tuck and J.A.K. Scott, editors, Engineering Mathematics and Applications Conference: EMAC98, pages 387-390, 1998. C1160, C1163

[6] E.M. Murman. Analysis of embedded shock waves calculated by relaxation methods. AIAA J., 12:626-633, 1974. C1160

[7] P.L. Roe. Approximate Riemann solvers, parameter vectors, and difference schemes. J. Computational Physics, 43:357-372, 1981. C1164

[8] E.F. Toro. Riemann Solvers and Numerical Methods for Fluid Dynamics. Berlin, 1997. C1163, C1168, C1168, C1171, C1172

[9] B. Van Leer. Towards the ultimate conservative difference scheme iv. a new approach to numerical convection. Journal of Computational Physics, 23:276-299, 1977. C1168

[10] B. Van Leer. Towards the ultimate conservative difference scheme v. a second-order sequel to Godunov's method. Journal of Computational Physics, 32:101-136, 1979. C1168 